\title{
Zusammenarbeit unter Anerkennung unserer jeweiligen Kompetenzen
}

\section{Michel Matter}

Dr. med., Vizepräsident der FMH, Departementsverantwortlicher Dienstleistungen und Berufsentwicklung

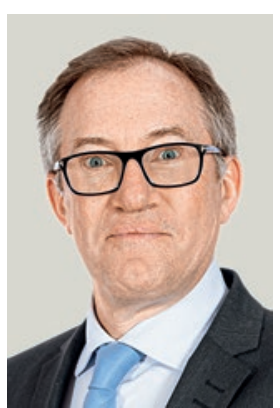

Im Vorwort zu den «Langfristperspektiven der öffentlichen Finanzen der Schweiz 2021» unterstreicht Ueli Maurer, Bundesrat und Vorsteher des Eidgenössischen Finanzdepartements, den demografischen Druck, der in den nächsten Jahren auf uns zukommen wird, und die damit einhergehenden finanziellen Anstrengungen in den Bereichen Gesundheit und Altersvorsorge. Die Herausforderung ist enorm, die Reformen sind unumgänglich. Neben dem Klimawandel muss die Alterung der Bevölkerung in politischer wie finanzieller Hinsicht zu einer der Prioritäten werden. Die Rolle der Pflegekräfte wird an Bedeutung gewinnen. An dieser Stelle muss unser Gesundheitssystem verteidigt und nicht angegriffen werden, wie es der Bund mit seinem Globalbudget vorhat. Die Versorgung zunehmend aktiverer Senioren, die hohe Ansprüche an ihre Lebensqualität und Akutversorgung stellen, auf der einen Seite sowie die zunehmend später erfolgende Begleitung am Lebensende auf der anderen Seite werden Paradigmenwechsel und Veränderungen der medizinischen Kultur erzwingen. Echte Interprofessionalität und damit eine deutlich verbesserte Koordination der Versorgung werden unumgänglich.

Echte Interprofessionalität und damit eine deutlich verbesserte Koordination der Versorgung werden unumgänglich.

Die paramedizinischen Berufe haben sich in den letzten Jahren weiterentwickelt, spezialisiert und an Kompetenz, Autonomie und Verantwortung gewonnen. Dies ist erfreulich und zudem unerlässlich, um die durch den demografischen Wandel gestellten Herausforderungen bewältigen zu können. Es gilt, die Kommunikation zwischen sämtlichen Akteuren des Gesundheitswesens zu verbessern. Zwingend erforderlich ist das - sehnlichst erwartete - gemeinsam genutzte elektronische Patientendossier, das relevante und sachdienliche medizinische und paramedizinische Informationen enthält und sämtliche elektronischen Systeme abdeckt, die derzeit in medizinischen Zentren und Arztpraxen im Einsatz sind. Wir müssen besser kommunizieren, die verschiedenen Meinungen und Bewertungen besser zusammenführen, besser koordinieren. Was so offensichtlich zu sein scheint, kommt kaum voran.

Interprofessionalität muss in der Ausbildung frühzeitig durch übergreifende Lehrpläne vermittelt werden, bei denen die gemeinsame Versorgung des Patienten oder der Patientin im Mittelpunkt steht. Interprofessionalität kann aber nur Realität werden, wenn sie sich

\section{Interprofessionalität muss in der Ausbildung} frühzeitig vermittelt werden und die gemeinsame Versorgung der Patienten im Mittelpunkt stehen.

in öffentlichen wie in privaten Praxen, in grossen wie in kleinen Pflegeeinrichtungen durchsetzt. Gemeinsam und unter Anerkennung unserer jeweiligen Kompetenzen werden wir diese Revolution, denn um nichts anderes handelt es sich, zum Erfolg führen. Ein solches Streben nach Effizienz und Effektivität muss auf unserer eigenen Tagesordnung stehen. Dies ist namentlich eine strategische Priorität des Departements Dienstleistungen und Berufsentwicklung der FMH, das sich in diesem Bereich stark engagiert.

Wohlgemerkt betrifft dies nicht alle Patientinnen und Patienten, aber doch eine grosse Anzahl. Manche Beobachter werden die Gefahr einer Kostenexplosion im Gesundheitswesen heraufbeschwören. Dem lässt sich leicht entgegenhalten, dass die ja vorhersehbare und somit Planungen ermöglichende Alterung der Bevölkerung eine politische und finanzielle Reaktion nach sich ziehen muss. Es müssen aber auch neue Wege zur Verbesserung der Patientenversorgung und zur Erzielung von Grössenvorteilen eingeschlagen werden. Wir alle haben unsere eigenen, ganz besonderen Fähigkeiten. In unseren jeweiligen Fachgebieten kümmern wir uns um die Patientinnen und Patienten und deren klinische Situation unter aktiver Mitwirkung aller anderen Gesundheitsberufe, wobei das angestrebte Ziel klar festgelegt ist.

Gemeinsam und mit Respekt. 